\title{
O processo da autorregulação no desenvolvimento de crianças
}

\author{
The self-regulation process on \\ child development
}

\author{
Maria Beatriz Martins LINHARES ${ }^{1}$ \\ Carolina Beatriz Savegnago MARTINS ${ }^{2}$
}

\section{Resumo}

A autorregulação é um processo básico relacionado aos desfechos adaptativos ao longo do desenvolvimento. O objetivo do presente artigo foi realizar uma revisão temática sobre o conceito de autorregulação e suas relações com o desenvolvimento da criança. Primeiramente, foram abordados os fundamentos teóricos sobre o processo de autorregularão no desenvolvimento, de acordo com o Modelo Transacional do Desenvolvimento de Arnold Sameroff. Em seguida, foi focalizada a caracterização deste processo de acordo com os seguintes componentes principais: a regulação cognitiva e os mecanismos neurocognitivos, a regulação emocional e a regulação comportamental. Além disso, foi tratada a relação entre os processos regulatórios e o temperamento. Finalmente, foi também analisada a relevância do papel dos correguladores na composição do processo autorregulatório no desenvolvimento da criança.

Palavras-chave: Autorregulação; Cognição; Comportamento; Personalidade.

\begin{abstract}
The self-regulation is a basic process related to adapted developmental outcomes. The aim of the present study was to present a topical review about the concept of self-regulation throughout the child development. First, the self-regulation of process of children was examined by Sameroff's Transactional Model of Development theoretical framework. Subsequently, the self-regulation process was characterized according to the following main components: cognitive regulation and neurocognitive mechanisms, emotional regulation, and behavioral regulation. Furthermore, the relationship between the regulatory processes and temperament was examined. Finally, the relevant influence of the co-regulator caregivers on composition of the self-regulatory process of child development was also analyzed.
\end{abstract}

Keywords: Self-regulation; Cognition; Behavior; Personality.

$\checkmark \nabla \nabla v$

1 Universidade de São Paulo, Faculdade de Medicina de Ribeirão Preto, Departamento de Neurociências e Ciências do Comportamento. Av. Tenente Catão Roxo, 2650, Campus Universitário Monte Alegre, 14051-140, Ribeirão Preto, SP, Brasil. Correspondência para/ Correspondence to: M.B.M. LINHARES. E-mail:<linhares@fmrp.usp.br>.

2 Universidade de São Paulo, Hospital das Clínicas da Faculdade de Medicina de Ribeirão Preto, Programa de Aprimoramento Profissional em Psicologia do Desenvolvimento na Área da Saúde. Ribeirão Preto, SP, Brasil.

Apoio: Conselho Nacional de Desenvolvimento Científico e Tecnológico (Processo n 301247/2010-2), Fundação de Amparo à Pesquisa do Estado de São Paulo (Processo n 2012/51633-0) e Fundação do Desenvolvimento Administrativo. 
O presente artigo trata-se de uma revisão temática sobre o conceito de autorregulação no desenvolvimento de crianças. Primeiramente, serão abordados alguns fundamentos teóricos sobre $\mathrm{o}$ processo de autorregulação no desenvolvimento, de acordo com o Modelo Transacional do Desenvolvimento de Sameroff (2009). Em seguida, será focalizada a caracterização deste processo de acordo com os seus principais componentes, a saber: a regulação cognitiva e os mecanismos neurocognitivos, a regulação emocional e a regulação comportamental. Além disso, será tratada a relação entre os processos regulatórios e temperamento, e finalmente será analisada a relevância do papel dos correguladores na construção do processo autorregulatório no desenvolvimento da criança.

\section{Fundamentos teórico-conceituais do processo de autorregulação}

Considerando-se a fundamentação teórica-conceitual de base para a compreensão dos processos regulatórios, o Modelo Transacional de Desenvolvimento, proposto por Sameroff (2009), trouxe uma grande contribuição devido a sua perspectiva desenvolvimental. Este modelo preconiza uma integração dialética no desenvolvimento humano entre as forças nature e nurture, incluindo os seguintes componentes: a pessoa, o contexto, a regulação e a representação (Sameroff, 2010). A pessoa inclui a progressão de competências sensório-motoras, cognitivas, afetivas e sociais, e os desempenhos nessas áreas. Por sua vez, o contexto integra diferentes níveis de sistemas ambientais e as influências bidirecionais entre a pessoa e diversos contextos, que incluem desde o microcontexto familiar até o macrocontexto da cultura em que a pessoa se insere. Nesta perspectiva, a regulação inclui processos que possibilitam atingir a autorregulação, por meio da mediação e da corregulação de outras pessoas, especialmente os cuidadores principais das crianças. Finalmente, a representação significa que as experiências das pessoas correspondem a representações no âmbito cognitivo-abstrato, que permitem interpretar novas experiências fu-

282 turas; além disso, permite formar esquemas de inter- pretação que têm função adaptativa, produzindo um conjunto de expectativas sobre como funciona o mundo à sua volta.

Pode-se destacar que, na pessoa, há integração das características biológicas (e.g., genética, idade, sexo) e psicológicas (temperamento, estados emocionais). Estas, por sua vez, interagem com os contextos sociais de desenvolvimento visando a uma auto-organização adaptativa. De acordo com este modelo, ocorrem diferentes processos regulatórios, a saber: a) as microrregulações, que incluem as interações face a face, momentâneas, entre a criança e seus cuidadores principais; b) as minirregulações, que ocorrem no contexto proximal familiar, incluindo as atividades de rotina diária; c) finalmente, as macrorregulações, que envolvem mudanças relevantes na experiência, com características mais duradouras, com forte determinação de fatores socioculturais (Sameroff \& Fiese, 2000).

O modelo transacional equilibra os efeitos bidirecionais entre pessoa e contexto e atribui um destaque para as interações experimentadas no contexto social familiar (Sameroff \& Fiese, 2000). Estas devem ser promotoras e protetoras do desenvolvimento da criança. Por outro lado, quando a criança vive em um ambiente familiar de caos, este interfere no fluxo de informações necessárias à autorregulação e corregulação promovida pelo outro, afetando, dessa forma, o desenvolvimento da criança (Sameroff, 2010). De acordo com a abordagem do Caos e sua influência no desenvolvimento (Evans \& Wachs, 2010), os contextos caóticos são caracterizados por ambientes físicos e humanos de grande instabilidade diária, sem ordem e falta de regularidade temporal e estrutural. Nesses ambientes, predominam, no microssistema, a variabilidade de cuidadores, a alta densidade de pessoas no contexto, o barulho, a multiplicidade de arranjos sem consistência no tempo, a alta complexidade visual, a excessiva estimulação. Essas condições podem afetar diretamente os processos de desenvolvimento regulatórios do indivíduo que está exposto a este tipo de ambiente (Wachs \& Evans, 2010).

A abordagem transacional sistêmica do desenvolvimento, proposta por Sameroff e Chandler 
(1975), caracteriza-se, portanto, pela integração e indissociação entre a pessoa e seus contextos de desenvolvimento, incluindo os processos intrapsicológicos e as ações em contextos, tanto interpessoais quanto físicos. Essas interrelações entre pessoa e contexto, envolvendo ações e reações, devem ser entendidas dentro de uma perspectiva temporal e dinâmica. Portanto, nesse processo transacional, o sistema de relações pode evoluir e modificar-se ao longo do tempo, a fim de a criança progressivamente atingir a autorregulação comportamental.

\section{Definição de autorregulação}

Os processos regulatórios no desenvolvimento ocorrem de forma hierarquizada, a fim de atingir uma maior organização, complexidade e intencionalidade (Feldman, 2009; Klein, Gaspardo, \& Linhares, 2011; Klein, Gaspardo, Martinez, Grunau, \& Linhares, 2009; Olson, Sameroff, Lunkenheimer, \& Kerr, 2009; Rothbart \& Bates, 2006; Sameroff, 2009). De acordo com esses autores, nos processos regulatórios iniciais, durante a fase neonatal, ocorre predominantemente a regulação fisiológica, que inclui, por exemplo, o ciclo de vigília e sono, e o controle de temperatura corporal. Em seguida, até 24 meses de idade, a criança adquire a regulação emocional, na qual é importante atingir o controle de emoções básicas, como o medo e a raiva. Entre 3 e 4 anos de idade, com os avanços dos processos de atenção (focalização e transferência da atenção, por exemplo) no desenvolvimento da criança, emerge a regulação do comportamento. Esta pode ser identificada na inibição de impulsos e pela habilidade de adiar gratificações. Portanto, nesta trajetória de organização, nota-se que, na fase pré-escolar, ocorre o surgimento do processo autorregulatório no desenvolvimento das crianças. Este integra diferentes processos regulatórios, que vão favorecer sua adaptação emocional e comportamental, considerando as demandas internas e externas ao organismo.

Define-se, assim, a autorregulação como a habilidade de monitorar e modular a emoção, a cognição e o comportamento, para atingir um obje- tivo e/ou adaptar às demandas cognitivas e sociais para situações específicas (Sroufe, 1995). Do controle externo passa a haver uma regulação interna, decorrente do processo de internalização e controle voluntário mediado pelo próprio indivíduo. De modo interessante, verifica-se que, desde muito cedo no desenvolvimento, as crianças protegem-se do excesso de ativação ou estimulação (Kopp, 1982). Como exemplo, pode-se identificar a busca por regulação do organismo nos seguintes indicadores: pegar a chupeta ou sugar o dedo para se acalmar, desviar o olhar, virar cabeça, fechar os olhos ou distrair-se, a fim de proteger-se da fonte de estresse ou de um estímulo novo/estranho; buscar colo ou consolo (aconchego).

\section{Componentes da autorregulação}

No desenvolvimento do processo de autorregulação, existe uma convergência e integração de processos regulatórios dos seguintes tipos: regulação cognitiva, regulação emocional e regulação comportamental (Berger, 2011; Vohs \& Baumeister, 2011). Nota-se, portanto, que diferentes áreas do desenvolvimento humano estão envolvidas na autorregulação. Além disso, o fator do temperamento, relativo ao controle voluntário, também contribui para o desenvolvimento do processo autorregulação (Rothbart, 2011). Os componentes do processo de autorregulação serão tratados a seguir:

\section{Regulação cognitiva}

A regulação cognitiva, que pode também ser denominada de "controle cognitivo", consiste na habilidade de reter mentalmente e manipular informação (memória de trabalho) e na habilidade de resistir à tentação de fazer algo (controle inibitório) (Paris \& Newman, 1990). O funcionamento executivo inclui uso de reflexão, competência e independência em completar tarefas e resolver problemas.

A regulação cognitiva envolve, portanto, relevantes mecanismos neurocognitivos, a saber: 
atenção, inibição, compartilhamento de tarefas e memória de trabalho. A atenção é a base do controle inibitório, da estratégia de resolução de problemas e do automonitoramento, envolvendo orientação, alerta, atenção sustentada e seleção de aspectos relevantes ou irrelevantes (Rueda, Posner, $\&$ Rothbart, 2011). A inibição envolve a função executiva, que reflete a habilidade de deliberadamente inibir uma resposta dominante automática ou preponderante (Miyake, Friedman, Emerson, Witzki, \& Howeter, 2000). O compartilhamento de tarefas corresponde à atenção executiva, que consiste na capacidade de dividir a atenção entre tarefas em andamento e o estado mental vinculado à outra tarefa (Miyake et al., 2000). Este mecanismo torna a pessoa capaz de lidar com mudanças nas demandas e nos objetivos de diferentes tarefas.

Na regulação cognitiva, a habilidade de focalizar na atividade (bom controle inibitório e boa capacidade de prestar atenção) está associada ao bom desempenho escolar (Blair \& Razza, 2007). Além disso, o controle com esforço/voluntário da atenção associa-se também ao comportamento social apropriado em situações com desafios emocionais (Kieras, Tobin, Graziano, \& Rothbart, 2005).

O estudo de Wang, Deater-Deckard, Petrill e Thompson (2012) exemplifica esse processo de regulação da atenção associado aos problemas de comportamento externalizante (agressão e delinquência) apresentados por crianças gêmeas vivendo situação de risco ambiental (lar caótico), dos 6 aos 8 anos de idade. Os professores avaliaram as dificuldades de atenção das crianças por meio do Teacher Report Form (Achenbach, 1991), considerando quando a criança falhava em terminar as tarefas, não se concentrava ou não prestava atenção por um longo tempo, e era desatenta ou facilmente distraída. Além disso, as crianças foram avaliadas em dois itens da Escala de Comportamento da Bayley, relativos à tarefa de atenção e à de persistência na tentativa de completar tarefas, por meio da observação de avaliadores treinados. A regulação da atenção foi medida, portanto, por um escore composto, que incluiu os três itens avaliados pelos professores e os dois itens de classificação do ava-

284 liador. Os resultados mostraram que, quanto maior o nível de caos ambiental, as crianças apresentam mais problema na regulação da atenção e mais problemas de comportamento externalizante na fase escolar.

O componente de função executiva no processo de autorregulação foi examinado em dois estudos descritos a seguir. O estudo de Kim-Spoon, Haskett, Longo e Nice (2012) examinou a autorregulação de crianças com histórico de abuso físico, na pré-escola, jardim de infância e $1^{\circ}$ ano, analisando os comportamentos parentais associados aos comportamentos externalizantes e internalizantes das crianças. A autorregulação foi avaliada a partir da percepção dos pais utilizando o Behavior Rating Inventory of Executive Function (Gioia, Isquith, Guy, \& Kenworthe, 2000). Os resultados mostraram que a dificuldade de autorregulação das crianças com história de abuso físico foi o único fator de vulnerabilidade significativo para o problema de comportamento externalizante na fase pré-escolar, e internalizante na fase de jardim de infância. Verificou-se, porém, que, quando as crianças com níveis mais baixos de autorregulação tinham pais com menores níveis de parentalidade positiva, essas apresentaram mais problemas de comportamento externalizante. Por outro lado, quando havia parentalidade positiva, ocorria um efeito moderador do baixo nível de autorregulação, diminuindo o nível dos problemas de comportamento externalizante.

O outro estudo analisou o grau da expressividade emocional dos pais e a autorregulação das crianças, com idade entre quatro e sete anos. Estas variáveis foram examinadas como preditores do comportamento escolar em crianças vítimas de abuso físico (Haskett, Stelter, Proffit, \& Nice, 2012). Na autorregulação, foi analisada a função executiva e a regulação emocional/comportamental das crianças pelo Behavior Rating Inventory of Executive Functioning (Gioia et al., 2000), de acordo com os relatos dos pais e professores. Verificou-se que a expressão parental de emoções negativas-submissas foi preditora do comportamento agressivo das crianças, revelando o papel mediador deste tipo de expressão na autorregulação das crianças. Por outro lado, houve associação entre expressão de emoções positivas parentais e o comportamento competente 
das crianças em sala de aula, que foi mediado pelas habilidades de autorregulação das crianças, conforme relatado pelos professores.

\section{Regulação emocional}

Em torno do primeiro ano de idade, pode-se observar o desenvolvimento da habilidade de regular a expressão das emoções. A partir de seis meses de idade, a criança apresenta as seguintes emoções básicas: alegria, tristeza, surpresa, reserva, medo e raiva. A regulação emocional envolve as habilidades e estratégias que servem para manejar, modular, inibir e melhorar a ativação emocional, de modo a dar suporte à adaptação social e respostas não sociais (Calkins, 2009; Kopp, 1989).

A regulação da emoção foi estudada associada aos transtornos de humor em crianças de 5 a 9 anos (Roy et al., 2013). A expressividade emocional foi avaliada em crianças com frequentes explosões temperamentais (imparing temper outbursts), que apresentavam baixa autorregulação, em comparação a um grupo controle. A expressividade emocional foi observada em tarefas de jogos computadorizados, sendo caracterizada como: global, positiva (frente ao sucesso) e negativa (frente à falha). Verificou-se que, quanto à regulação emocional, as crianças com frequentes explosões temperamentais exibiam, em comparação ao grupo controle, pouca expressão positiva e menos regulação da expressividade negativa e expressão negativa frente à frustração. Foi também encontrado que, em $21,6 \%$ das crianças com grave desregulação do humor, as mães tinham desordens de ansiedade, o que interferia sobremaneira nos processos regulatórios das crianças.

O marco da autorregulação na fase pré-escolar envolve o controle do ego e a resiliência do ego, que são fatores que favorecem o ajustamento global (Causadias, Salvatore, \& Sroufe, 2012). A resiliência do ego foi forte preditora do funcionamento adaptativo, assim como de problemas de comportamento internalizante e externalizante na fase adulta. O conceito de controle de ego e resiliência do ego são constructos relevantes para compreender o desenvolvimento de autorregulação, ajusta- mento global e problema de comportamento (Causadias et al., 2012; Letzring, Block, \& Funder, 2005). O controle do ego é a capacidade de regular e expressar emoções e sentimentos, sendo que altos níveis de expressão emocional caracterizam pobre controle regulatório do ego em diferentes contextos; por outro lado, o supercontrole do ego envolve grande inibição, excesso de adiamento de gratificação e pouca experiência emocional. Crianças com pobre controle do ego desenvolveram mais problemas de comportamento internalizante e externalizante na fase da adolescência (Causadias et al., 2012; Chuang, Lamb, \& Hwang, 2006). Por outro lado, alto nível de resiliência do ego promove alto controle do ego e funcionamento adaptativo no desenvolvimento (Causadias et al., 2012; Chuang et al., 2006). A resiliência do ego, por sua vez, é um elemento essencial para proteção de amostras de risco (pobreza, baixa renda, ter mãe solteira e baixo nível educacional das mães) (Causadias et al., 2012; Klohnen, Vandwaler, \& Young, 1996).

\section{Regulação comportamental}

A regulação comportamental se caracteriza pela habilidade de manejar ou controlar seu próprio comportamento, que inclui: obedecer às demandas e direções dos adultos; controlar respostas impulsivas; adiar engajar-se em atividades específicas (Kochanska, Murray, \& Coy, 1997; Kopp, 1982). No dia a dia, pode-se observar a regulação comportamental, por exemplo, quando a criança consegue sentar para ouvir histórias ou andar sem correr. A regulação comportamental no início do desenvolvimento está associada a competência e habilidades sociais posteriormente (Eisenberg, Fabes, Gunthrie, \& Reiser, 2000). Portanto, deve-se observar os padrões de autorregulação inicial, que fornecem a base dos padrões individuais da autorregulação ao longo do desenvolvimento (Carlson, Sroufe, \& Egeland, 2004; Causadias et al., 2012).

De acordo com Kochanska e Aksan (2006), a consciência da criança e a emergência da autorregulação dependem de dois sistemas iniciais inibitórios do temperamento, ou seja, medo e controle com esforço/voluntário, e da qualidade das intera- 
ções com os pais no processo de socialização. O temperamento se refere a diferenças individuais, relativamente estáveis ao longo do desenvolvimento, incluindo fatores tanto biológicos quanto psicológicos, formando a base da personalidade (Rothbart, 2011). O temperamento consiste em diferenças individuais, com base constitucional, na reatividade e autorregulação, nos domínios do afeto, atividade e atenção. $O$ termo constitucional refere-se às bases relativamente biológicas do temperamento, as quais são influenciadas pela hereditariedade, maturação e experiência. A reatividade refere-se às características da responsividade individual a mudanças de estimulação externa ou interna. A autorregulação, por sua vez, consiste nos mecanismos usados pelo indivíduo para controlar suas reações emocionais e comportamentais frente a fontes de estimulação positiva ou negativa.

O temperamento inclui três grandes fatores, a saber: afeto negativo, extroversão e controle com esforço (Rothbart, 2011). Esses fatores incluem diferentes dimensões, a saber: a) afeto negativo, que inclui dimensões do tipo desconforto, medo, frustração, tristeza e capacidade de se acalmar (escore invertido); b) extroversão, que é composto por dimensões do tipo de prazer de alta intensidade, nível de atividade, impulsividade; c) controle com esforço/ voluntário, que é composto por dimensões do tipo de controle inibitório, focalização da atenção e prazer de baixa intensidade (Putnam, Ellis, \& Rothbart, 2001; Putnam, Gartstein, \& Rothbart, 2006).

As formas iniciais do afeto negativo incluem formas primitivas de irritação, angústia, seguidas por estados mais organizados relacionados à frustração e ao medo (Bridgett et al., 2009). A estrutura do afeto negativo tem demonstrado relativa constância ao longo do ciclo de vida, de acordo com as medidas desse fator nas fases de bebê, primeira infância, segunda infância (fase escolar) e adolescência, geralmente incluindo dimensões de medo, raiva e tristeza (Putnam et al., 2001). Em relação às outras características, o afeto negativo é um dos primeiros aspectos do temperamento a surgir no desenvolvimento do indivíduo (Bridgett ciação rápida de resposta, alto nível de atividade, preferência por situações caracterizadas por estímulos de alta intensidade (exposição a riscos) e relativa inquietação em novas situações sociais (Rothbart, 2011). O fator controle com esforço reúne traços que parecem ter em comum a regulação, voluntária e intencional, da criança sobre a atenção e o comportamento. O controle com esforço refere-se à capacidade de inibir uma resposta dominante para realizar uma resposta subdominante (Rothbart, Ahadi, Hershey, \& Fisher, 2001). Este fator é em grande parte um produto do desenvolvimento do sistema de atenção executiva (Rothbart, 2004), e desempenha um papel central nos processos de socialização da criança (Rothbart \& Bates, 2006).

O temperamento da criança exerce forte influência na emergência de comportamentos desajustados e indicadores de psicopatologia em crianças. Durante os anos pré-escolares, foram encontradas relações diretas entre fatores do temperamento e tipos específicos de problemas emocionais e de comportamentos internalizantes e externalizantes (Cosentino-Rocha \& Linhares, 2013; Gracioli \& Linhares, 2014; Linhares, Dualibe, \& Cassiano, 2013). Os problemas de comportamentos externalizantes relacionam-se com maior nível de atividade (De Pauw, Mervielde, \& Leeuwen, 2009), menor controle com esforço (De Pauw et al., 2009; Schlotz, Jones, Godfrey, \& Phillips, 2008; Zhou et al., 2008), maior extroversão (Berdan, Keane, \& Calkins, 2008; De Pauw, Mervielde, Van Leeuwen, \& De Clercq, 2011), maior raiva e frustração (Zhou et al., 2008) e maior afeto negativo (De Pauw et al., 2011). Os problemas de comportamento internalizantes, por sua vez, estavam associados a maior afeto negativo (De Pauw et al., 2009; 2011) e menor controle com esforço (De Pauw et al., 2011).

O temperamento com elevado traço de afeto negativo (frustração, medo, tristeza, dificuldade para se acalmar, desconforto e sensibilidade perceptual) foi preditor de mais problemas de comportamento internalizante (Edgar et al., 2008). Por outro lado, um pobre controle com esforço, que envolve problemas de ter prazer de baixa intensidade, controle inibitório e focalização da atenção, 
foi preditor de mais problemas de comportamento do tipo externalizante (Prior et al., 2008). Essas crianças com problemas de comportamento externalizante estão em risco de rejeição pelos companheiros, conflitos com a família e com os professores, fracasso escolar, dificuldades ocupacionais, além do risco mais sério para comportamentos socialmente desviantes (Olson et al., 2009). Deve-se destacar, no entanto, que o controle com esforço envolve um processo de regulação do afeto negativo e, portanto, afeta o processo de autorregulação, que influencia a adaptação da criança (Calkins, 2009).

O controle com esforço/voluntário foi um indicador importante da habilidade de autorregulação, medido por atraso de gratificação, em crianças com idade média de 5 anos (Martin, Razza, \& Brooks-Gunn, 2011). A medida do atraso de gratificação envolveu a tarefa do presente embrulhado (gift wrap) de Kochanska, Murray e Harlan (2000). Os achados mostraram que as crianças que tinham dificuldade de controle voluntário em adiar gratificação eram provenientes de lares caóticos, com falta de rotina e pobre vocabulário receptivo. Nesse sentido, as crianças da fase pré-escolar, com pobre processo regulatório no desenvolvimento, não contavam com a organização e estimulação adequada no contexto ambiental familiar.

O controle com esforço/voluntário do temperamento, avaliado ao longo do desenvolvimento até à fase escolar, mostrou ser um importante fator de autorregulação desenvolvimental. No estudo de Choe, Olson e Sameroff (2013), a autorregulação foi avaliada pela bateria de Kochanska, Murray, Jaques, Koenig, \& Vandegeest (1996) e Kochanska et al. (1997), aos 3 e 6 anos de idade, e obtido um índice de autorregulação. Verificou-se que crianças educadas com disciplina parental indutiva apresentaram alto nível de autorregulação, medida pelo controle com esforço, aos 3 anos. Este, por sua vez, predisse menos problemas de comportamento posteriormente, aos 6 anos de idade, de acordo com relato materno. Destaca-se também que, quando, além da disciplina parental indutiva, as crianças eram procedentes de famílias com nível socioeconômico mais alto e tinham alto controle com esforço aos 3 anos de idade, elas apresentavam posteriormente melhor controle com esforço aos 6 anos de idade. Por outro lado, quando havia pobre autorregulação aos 3 anos, as crianças tinham mais problemas de comportamento externalizante entre 6 e 10 anos. Nesse sentido, esses indicadores autorregulatórios parecem ser fatores protetores do desenvolvimento, reduzindo os problemas comportamentais da criança nas fases pré-escolar e escolar.

\section{O papel dos correguladores}

Nos diferentes processos de regulação apresentados até o momento, verifica-se a presença relevante dos pais e cuidadores como correguladores de todos esses processos. Há fortes evidências anteriores de que a interação com adultos mais desenvolvidos e capazes do que a criança consiste em elemento fundamental para o desenvolvimento da mediação social adequada, que, por sua vez, promove o desenvolvimento da criança (Sameroff, 2009; Vygotsky, 1996). Além disso, os pais que tinham temperamento com mais controle com esforço/voluntário apresentaram melhores práticas parentais e mais suporte às crianças (Eisenberg, Clumberland, \& Spinrad, 1998). Verificou-se também que o temperamento materno com menos controle inibitório foi preditor de problemas de comportamento em crianças nascidas prematuras, na fase de 18-36 meses (Gracioli, 2013).

Assim, o processo de ativação e regulação psicobiológica do temperamento é uma importante característica da pessoa, que pode atuar como variável moderadora de processos de socialização e padrões de comportamento das crianças (Bates, Goodnight, Fite, \& Staples, 2009). O temperamento de crianças também tem forte associação e interação com características dos cuidados parentais e práticas educativas, reforçando a perspectiva transacional no desenvolvimento da criança (Sameroff, 2009), que postula a reciprocidade, a sincronia e a responsividade nas interações entre pais e crianças.

As práticas educativas parentais podem ser efetivas para as regulações emocionais e comportamentais. A relação entre temperamento da criança com alto controle inibitório foi preditor de trans- 
torno de ansiedade; porém, esta relação tornava-se mais acentuada quando a mãe apresentava ansiedade e práticas educativas superprotetoras (Hudson, Dodd, \& Lyneham, 2009). Especialmente em populações de risco social, a emoção materna relacionada com a socialização desempenha um papel importante na regulação de crianças (Brophy-Herb, Stansbury, Brocknek, \& Horodynski, 2012). Por outro lado, mães que apresentavam um temperamento com maior controle com esforço e permaneciam mais tempo com a criança tinham impacto positivo no aumento do controle com esforço no temperamento de crianças de 18 meses de idade (Brigget et al., 2011).

Portanto, práticas educativas parentais contribuem para a regulação emocional das crianças. Verificou-se que tentativas maternas de mudar o foco de atenção da criança durante uma frustração associaram-se com menor expressão de raiva e tristeza por parte das crianças na idade pré-escolar e escolar (A.S. Morris, Morris, Silk, \& Steinberg, 2011). Além disso, nesse estudo, foi encontrado que a estratégia de reenquadramento cognitivo conjunto, na qual as díades mãe-criança estavam envolvidas, também foi associada com menor expressão de raiva e tristeza das crianças.

As estratégias parentais indulgentes apresentaram-se associadas a comportamentos agressivos nas crianças com baixo ou moderado controle com esforço e alta ou moderada busca por sensações, mas não em crianças com alto controle com esforço e moderada e baixa busca por sensações. Por outro lado, estratégias parentais coercitivas mostraram associações com agressividade nas crianças com alta ou moderada busca por sensações, mas não nas crianças com baixa busca por sensações (Xu, Farver, \& Zhang, 2009). Como evidência de que a reatividade da criança também pode ter impacto nos pais, o estudo de Burney e Leerkes (2010) mostrou que havia maior coparentalidade, em que os parceiros dividiam tarefas de cuidados parentais, quando os bebês apresentavam maior capacidade de se acalmar.

Os estilos parentais também influenciam o processo de autorregulação no desenvolvimento da 288 criança. Entre esses estilos, o comportamento intru- sivo parental constitui-se em um fator de risco para o desenvolvimento de habilidades de autorregulação e sociais em crianças com atraso no desenvolvimento, com pontuação igual ou menor do que 85 na Escala Mental de Bayley aos 3 anos (Stevenson \& Crnic, 2013). A regulação foi avaliada por meio das reações comportamentais das crianças, incluindo duração, intensidade, frequência e labilidade, frente às demandas da realização de uma tarefa, no caso uma montagem de quebra-cabeça (Cole, Michel, \& Teti, 1994). A intrusividade do pai, independentemente da intrusividade materna e dos problemas de comportamento da criança, relacionaram-se diretamente com poucas habilidades sociais das crianças, mediada pelo seu próprio comportamento com pouca regulação. Nota-se, portanto, que o estilo intrusivo paterno é um importante fator de risco para o desenvolvimento do processo regulatório e a competência social em crianças com atraso na área de desenvolvimento cognitivo. Por outro lado, os estilos parentais com autoridade, disciplina assertiva e métodos indutivos ajudam no processo de internalização, controle individual, independência e sociabilidade das crianças. Os pais agressivos ou intrusivos prejudicam a autonomia das crianças (Baumrind, 1972).

A relação entre cuidados parentais e o surgimento da autorregulação, em crianças de 2 anos e 6 meses, de famílias de baixa renda e de minorias étnicas (afroamericana e latina), foi examinada por Owen et al. (2013). A autorregulação foi avaliada pelas seguintes tarefas de inibição comportamental simples (Kochanska et al., 2000), a saber: atraso do lanche, presente embrulhado e brinquedo proibido. Foi também analisada uma tarefa de inibição comportamental complexa, que envolvia um desafio para resolução de conflitos pela criança. Os cuidados parentais foram examinados por meio de observação direta da interação das mães e pais com a criança, e questionário sobre envolvimento do pai. Verificou-se que melhores habilidades de regulação comportamental das crianças, envolvendo resposta de inibição simples, foram consistentemente preditas apenas pelos cuidados maternos orientados para criança (child-oriented mothering). Os cuidados maternos que favoreceram a emer- 
gência da autorregulação envolviam sensibilidade, cuidado caloroso e estimulação, em ambas as etnias. A qualidade dos cuidados do pai (child-oriented fathering), por sua vez, foi preditora das respostas inibitórias complexas apenas nas crianças de famílias afroamericanas. A tarefa de inibição complexa envolvia um desafio com resolução de conflitos. Esses achados reforçam o efeito diferencial dos cuidados de ambos os pais no processo regulatório comportamental no desenvolvimento de crianças provenientes de minorias étnicas.

Em idade mais avançada, o estudo de Piotrowski, Lapierre e Linebarger (2013) teve como objetivo investigar as variáveis associadas com o comportamento autorregulatório de crianças entre 5 a 9 anos de idade. A autorregulação foi avaliada por meio de três subescalas do sistema de avaliação Behavioral Assessment System for Children - 2 de avaliação do comportamento para crianças (Reynolds \& Kampaus, 2004), de acordo com a percepção dos pais, que incluíam itens sobre a habilidade das crianças de regulação comportamental e cognitiva. Os problemas autorregulatórios relacionaram-se com as seguintes variáveis: a presença de alguma deficiência no desenvolvimento da criança, crianças de baixa renda, mais novas (entre 5-9 anos de idade), do sexo masculino e não brancas ou latinas, morando no contexto norte-americano (Piotrowski et al., 2013). De forma interessante, ainda neste estudo, verificou-se que, tanto o estilo parental permissivo, quanto o estilo autoritário foram associados com menos habilidade de autorregulação no desenvolvimento das crianças; por outro lado, as crianças de pais com o estilo educacional com autoridade tinham mais habilidades autorregulatórias.

Portanto, nota-se que a habilidade de corregulação dos pais contribui efetivamente para o processo de autorregulação no desenvolvimento das crianças, pois pode facilitar a internalização de princípios, regras e valores. Os programas de práticas educativas parentais facilitam a aquisição de habilidades regulatórias e o manejo do comportamento dos próprios pais e destes em relação às crianças. De acordo com Sameroff e Fiese (2000), entre as estratégias de intervenção educativas, a reeducação dos pais é fundamental. Permite desen- volver as competências parentais básicas para regular o comportamento das crianças, ensinando e promovendo estratégias e práticas educativas parentais adequadas.

Entre os programas efetivos para melhorar as práticas educativas parentais, pode-se destacar dois programas de prevenção universal, que se destacam na literatura, a saber: Triple - P - Positive Parenting Program (Sanders, 2008) e ACT - Raising Safe Kids Program (Silva, 2009). O primeiro - Triple $P$-, tem por objetivo ensinar práticas educativas efetivas para lidar com comportamentos disruptivos de crianças na fase pré-escolar. O programa visa a promover nos pais autossuficiência, autoeficácia automanejo para melhorar as práticas educativas de forma eficiente, para atender as necessidades da criança, por meio de disciplina assertiva, ajuste de expectativas e criação de um ambiente positivo de aprendizagem. Os pais aprendem a resolver problemas ao definir o problema e ao formular, executar e avaliar planos de mudanças. O segundo programa - ACT, por sua vez, tem por objetivo também melhorar as práticas educativas parentais e prevenir violência e maus tratos nos cuidados às crianças. É interessante destacar que, neste último programa, existem módulos específicos para os cuidadores aprenderem manejo da raiva dos próprios cuidadores e dos filhos, respectivamente. $\mathrm{O}$ ACT oferece estratégias para regular o sentimento de raiva, de forma a identificar sua própria raiva e a raiva da criança. Nesse sentido, o foco da autorregulação bidirecional é estimulada para facilitar os processos interacionais. As crianças pequenas são dependentes da regulação externa e gradualmente aprendem a autorregular.

Em suma, pode-se concluir que o processo autorregulatório no desenvolvimento tem sido investigado sob diferentes perspectivas e ênfases nos diversos domínios cognitivos, emocionais e comportamentais. Entretanto, na literatura científica, verificou-se que, apesar de haver diferentes definições acerca deste processo, que dependem do domínio em foco e das diferentes medidas do constructo de regulação, há um consenso sobre seu impacto e sua relevância nos processos adaptativos das crianças, em sua trajetória de desenvolvimento. 
As características das medidas e avaliações do constructo da autorregulação ajudam a definir operacionalmente e circunscrever o que se trata por autorregulação na literatura. Além disso, destaca-se que o papel dos cuidadores é essencial para compreender o processo autorregulatório no desenvolvimento das crianças, pois este permite moderar principalmente as regulações emocionais e comportamentais das crianças.

No entanto, deve-se destacar que, apesar de o presente estudo mostrar grandes contribuições sobre autorregulação e desenvolvimento da criança, este artigo tem certas limitações. O presente estudo não teve por objetivo realizar uma revisão sistemática dos estudos empíricos e nem esgotar todas as vertentes teórico-conceituais que abordam este tema. Estudos futuros podem avançar nesse sentido e em outros aspectos, a saber: na compreensão dos mecanismos subjacentes aos processos de regulação no desenvolvimento; no desenvolvimento de estudos longitudinais-prospectivos para acompanhar a evolução dos processos regulatórios ao longo das diferentes fases evolutivas; no estabelecimento do impacto diferencial das falhas nos diferentes processos regulatórios, respectivamente, e as diversas psicopatologias na infância.

\section{Referências}

Achenbach, T. M. (1991). Manual for the teacher's report form and 1991 profile. Burlington, VT: University of Vermont.

Bates, J. E., Goodnight, J. A., Fite, J. E., \& Staples, A. D. (2009). Behavior regulation as the product of temperament and environment. In S. Olson \& A. Sameroff (Eds.), Biopsychosocial regulatory processes in the development of childhood behavioral problem (pp.116-143). New York: Cambridge University Press.

Baumrind, D. (1972). The development of instrumental competence through socialization. In A. D. Pick (Ed.), Minnesota Symposia on Child Psychology (pp.3-46). Oxford: University of Minnesota Press.

Berdan, L. E., Keane, S. P., \& Calkins, S. D. (2008). Temperament and externalizing behavior: Social preferences and perceived acceptance as protective factors. Developmental Psychology, 44(4), 957-968. http://dx.doi.org/10.1037/0012-1649.44.4.957

Berger, A. (2011). Self-regulation: Brain, cognition, and development. Washington, DC: American Psychological
Blair, E., \& Razza, R. P. (2007). Relating effortful control, executive function, and false belief understanding to emerging math and literacy ability in kindergarten. Child Development, 78(2), 647-663, http://dx.doi.org/ 10.1111/j.1467-8624.2007.01019.x

Bridgett, D. J., Gartstein, M. A., Putnam, S. P., McKay, T., Iddins, E., Robertson, C., ... Rittmueller, A. (2009). Maternal and contextual influences and the effect of temperament development during infancy on parenting in toddlerhood. Infant Behavior and Development, 32(1), 103-116. http://dx.doi.org/ 10.1016/j.infbeh.2008.10.007

Bridgett, D. J., Gartstein, M. A., Putnam, S. P., Lance, K. O., Iddins, E., Waits, R., ... Lee, L. (2011). Emerging effortful control in toddlerhood: The role of infant orienting/regulation, maternal effortful control, and maternal time spent in caregiving activities. Infant Behavior and Development, 34(1), 189-199. http:// dx.doi.org/10.1016/j.infbeh.2010.12.008

Brophy-Herb, H. E., Stansbury, K., Bocknek, E., \& Horodynski, M. A. (2012). Modeling maternal emotion-related socialization behaviors in a low-income sample: Relations with toddlers' self-regulation. Early Childhood Research Quarterly, 27(3), 352-364. http:// dx.doi.org/10.1016/j.ecresq.2011.1 1.005

Burney, R. V., \& Leerkes, E. M. (2010). Links between mothers and fathers' perceptions of infant temperament and coparenting. Infant Behavior and Development, 33(2), 125-135. http://dx.doi.org/10.1016/j.infbeh. 2009.12.002

Calkins, S. (2009). Regulatory competence and early disruptive behavior problems: The role of physiological regulation. In S. L. Olson \& A. J. Sameroff (Eds.), Biopsychosocial regulatory processes in the development of childhood behavioral problems (pp.86-107). New York: Cambridge University Press.

Carlson, E. A., Sroufe, L. A., \& Egeland, B. (2004). The construction of experience: A longitudinal study of representation and behavior. Child Development, 75(1), 66-83. http://dx.doi.org/10.1111/j.1467-86 24.2004.00654.x

Causadias, J. M., Salvatore, J. E., \& Sroufe, L. A. (2012). Early patterns of self-regulation as risk and promotive factors in development: A longitudinal study from childhood to adulthood in a high-risk sample. International Journal of Behavioral Development, 36(4), 293-302. http://dx.doi.org/10.1177/016502 5412444076

Choe, D. E., Olson, S. L., \& Sameroff, A. J. (2013). Effects of early maternal distress and parenting on the development of children's self-regulation and externalizing behavior. Development and Psychopathology, 25(2), 437-453. http://dx.doi.org/10.1017/S095457941200 1162

Chuang, S. S., Lamb, M., \& Hwang, C. P. (2006). Personality development from childhood to adolescence: A longitudinal study of ego-control and ego-resiliency 
in Sweden. International Journal of Behavioral Development, 30(4), 338-343. http://dx.doi.org/ $10.1177 / 0165025406072795$

Cole P. M., Michel M. K., \& Teti L. O. (1994). The development of emotion regulation and dysregulation: A clinical perspective. Monographs of the Society for Research in Child Development, 59(2/3), 73-100. http:// dx.doi.org/10.2307/1166139

Cosentino-Rocha, L., \& Linhares, M. B. M. (2013). Temperamento de crianças e diferenças de gênero. Paidéia, 23(54), 63-72. http://dx.doi.org/10.1590/1982-432 72354201308

De Pauw, S. S. W., Mervielde, I., \& Leeuwen, K. G. V. (2009). How are traits related to problem behavior in preschoolers? Similarities and contrasts between temperament and personality. The Journal of Abnormal Child Psychology, 37(3), 309-325. http:// dx.doi.org/10.1007/s10802-008-9290-0

De Pauw, S. S., Mervielde, I., Van Leeuwen, K. G., \& De Clercq, B. J. (2011). How temperament and personality contribute to the maladjustment of children with autism. Journal of Autism Developmental Disorders, 41(2), 196-212. http://dx.doi.org/10.1007/s10803010-1043-6

Edgar, K. P., Schmidt L. A., Henderson, H. A., Schulkin, J, Fox, N. A., \& Feldman, R. (2008). Salivary cortisol levels and infant temperament shape developmental trajectories in boys at risk for behavioral maladjustment. Psychoneuroendocrinology, 33(7), 916-925. http:// dx.doi.org/10.1016/j.psyneuen.2008.03.018

Eisenberg, N., Clumberland, A. Z., \& Spinrad, T. L. (1998). Parental socialization of emotion. Psychological Inquiry, 9(4), 241-273. http://dx.doi.org/10.1207/s1532796 5pli0904_1

Eisenberg, N., Fabes, R. A., Gunthrie, I. K., \& Reiser, M. (2000). Dispositional emotionality and regulation: The role in predicting quality of social functioning. Journal of Personality and Social Psychology, 78(1), 136-157. http://dx.doi.org/10.1037/0022-3514.78.1.136

Evans, G. W., \& Wachs, T. D. (2010). Chaos and its influence on children's development: An ecological perspective. Washington, DC: American Psychological Association.

Feldman, R. (2009). The development of regulatory functions from birth to 5 years: Insights from premature infants. Child Development, 80(2), 544-61. http://dx.doi.org/10.1111/j.1467-8624.2009.01278.x

Gioia, G. A., Isquith, P. K., Guy, S. C., \& Kenworthy, L. (2000). Behavior rating inventory of executive functioning manual. Odessa, FL: Psychological Assessment Resource.

Gracioli, S. M. A. (2013). Temperamento e comportamento de crianças nascidas pré-termo extremo e moderado na fase de 18 a 36 meses (Dissertação de mestrado não-publicada). Universidade de São Paulo, Ribeirão Preto.
Gracioli, S. M., \& Linhares, M. B. M. (2014). Temperamento e sua relação com problemas emocionais e de comportamento em pré-escolares. Psicologia em Estudo, 19(1), 71-80. http://dx.doi.org/10.1590/14137372213020007

Haskett, M. E., Stelter, R., Proffit, K., \& Nice, R. (2012). Parent emotional expressiveness and children's selfregulation: Associations with abused children's school functioning. Child Abuse \& Neglect, 36(4), 296-307. http://dx.doi.org/10.1016/j.chiabu.2011.11.008

Hudson, J. L., Dodd H. F., \& Lyneham, H. J. (2009) Temperament and family environment in the development of anxiety disorder: Two-year follow-up. Journal of the American Academy of Child and Adolescent Psychiatry, 50(12), 55-64. http://dx.doi.org/10.1016/j.jaac.201 1.09 .009

Kieras, J. E., Tobin, R. M., Graziano, W. G., \& Rothbart, M. K. (2005). You can't always get what you want: Effortful control and children's responses to undesirable gifts. Psychological Science, 16(5), 391-396. http:// dx.doi.org/10.1111/j.0956-7976.2005.01546.x

Kim-Spoon, J., Haskett, M. E., Longo, G. S., \& Nice, R. (2012). Longitudinal study of self-regulation, positive parenting and adjustment problems among physically abused children. Child Abuse \& Neglect, 36(2), 95107. http://dx.doi.org/10.1016/j.chiabu.2011.09.016

Klein, V. C., Gaspardo, C. M., \& Linhares, M. B. M. (2011). Dor, autorregulação e temperamento em recémnascidos pré-termo de alto risco. Psicologia: Reflexão e Crítica, 24(3), 542-550. http://dx.doi.org/10.1590/ s0102-79722011000300011

Klein, V. C., Gaspardo, C. M., Martinez, F. E., Grunau, R. E., \& Linhares, M. B. (2009). Pain and distress reactivity and recovery as early predictors of temperament in toddlers born preterm. Early Human Development, 85(9), 569-576. http://dx.doi.org/10.1016/j.earlhum dev.2009.06.001

Klohnen, E. C., Vandewater, E. A., \& Young, A. (1996). Negotiating the middle years: Ego-resiliency and successful midlife adjustment in women. Psychology and Aging, 11(3), 431-442. http://dx.doi.org/10.1037/ 0882-7974.11.3.431

Kochanska, G., \& Aksan, N. (2006). Children's conscience and self-regulation. Journal of Personality, 74(6), 1587-1617. http://dx.doi.org/10.1111/j.1467-64 94.2006.00421.x

Kochanska, G., Murray, K. T., \& Coy, K. C. (1997). Inhibitory control as a contributor to conscience in childhood: From toddler to early school age. Child Development, 68(2), 263-277. http://dx.doi.org/10.2307/1131849

Kochanska, G., Murray, K. T., \& Harlan, E. T. (2000). Effortful control in early childhood: Continuity and change, antecedents, and implications for social developmental. Developmental Psychology, 36(2), 220-232. http://dx.doi.org/KM037//0012-1649.3 6.2 .220 
Kochanska, G., Murray, K. T., Jacques, T. Y., Koenig, A. L., \& Vandegeest, K. A. (1996). Inhibitory control in young children and its role in emerging internalization. Child Development, 67(2), 490-507. http://dx.doi.org/ $10.2307 / 1131828$

Kopp, C. B. (1982). Antecedents of self-regulation: A developmental perspective. Developmental Psychology, 18(2), 199-214. http://dx.doi.org/10.1037/0012-16 49.18.2.199

Kopp, C. B. (1989). Regulation of distress and negative emotions: An integrative review. Developmental Psychology, 25(3), 343-354. http://dx.doi.org/10.10 37/0012-1649.25.3.343

Letzring, T. D., Block J., \& Funder D. C. (2005). Ego-control and ego-resiliency: Generalization of self-report scales based on personality descriptions from acquaintances, clinicians, and the self. Journal of Research in Personality, 39(4), 395-442. http://dx.doi.org/10.1016/ j.jrp.2004.06.003

Linhares, M. B. M., Dualibe, A., \& Cassiano, R. M. (2013). Temperamento de crianças na abordagem de Rothbart: estudo de revisão sistemática. Psicologia em Estudo, 18(4), 633-645. http://dx.doi.org/10.1590/S1413-737 22013000400006

Martin, A., Razza, R. A., \& Brooks-Gunn, J. (2011). Specifying the links between household chaos and preschool children's development. Early Child Development and Care, 182(3-4), 1-17. http://dx. doi.org/10.1080/03004430.2011.605522

Miyake, A., Friedman, N. P., Emerson, M. J., Witzki, A. H., \& Howerter, A. (2000). The unity and diversity of executive functions and their contributions to complex "frontal lobe" tasks: A latent variable analysis. Cognitive Psychology, 41(1), 49-100. http://dx.doi.org/ 10.1006/cogp.1999.0734

Morris, A. S., Morris, M. D. S., Silk, J. S., \& Steinberg, L. (2011). The influence of mother-child emotion regulation strategies on children's expression of anger and sadness. Developmental Psychology, 47(1), 213-225. http://dx.doi.org/10.1037/a0021021

Olson, S. I., Sameroff, A., Lunkenheimer, E. S., \& Kerr, D. (2009). Self-regulatory processes in the development of disruptive behavior problems: The preschool to school transition. In S. L. Olson \& A. J. Sameroff (Eds.), Biopsychosocial regulatory processes in the development of childhood behavioral problems (pp.144-185). New York: Cambridge University Press.

Owen, M. T., Caughy, M. O., Hurst, J. R., Amos, M., Hasanizadeh, N., \& Mata-Otero, A. M. (2013). Unique contributions of fathering to emerging self-regulation in low-income ethnic minority preschoolers. Early Child Development Care, 183(3-4), 464-482. http://dx. doi.org/10.1080/03004430.2012.711594

Paris, S., \& Newman, R. (1990). Developmental aspects of self-regulated learning. Educational Psychologist, 25(1), 87-102. http://dx.doi.org/10.1207/s1532698
Piotrowski, J. T., Lapierre, M. A., \& Linebarger, D. L. (2013). Investigating correlates of self-regulation in early childhood with a representative sample of Englishspeaking American families. Journal of Child and Family Studies, 22(3), 423-436. http://dx.doi.org/ 10.1007/s10826-012-9595-z

Prior, M., Bavin, E. L., Cinic, E., Reillyc, S., Bretherton, L., Wake, L., \& Eadie, P. (2008). Influences on communicative development at 24 months of age: Child temperament, behavior problems, and maternal factors. Infant Behavior \& Development, 31(2), 270-279. http:// dx.doi.org/10.1016/j.infbeh.2007.11.001

Putnam, S. P., Ellis, L. K., \& Rothbart, M. (2001). The structure of temperament from infancy to adolescence. In A. Eliaz, \& A. Angleitner (Eds.), Advances in research of temperament (pp.165-182). Lingerich: Pabst Scientific.

Putnam, S. P., Gartstein, M. A., \& Rothbart, M. K. (2006). Measurement of fine-grained aspects of toddler temperament: The early childhood behavior questionnaire. Infant Behavior and Development, 29, 386-401. http://dx.doi.org/10.1016/j.infbeh.2006. 01.004

Reynolds, C. R., \& Kampaus, R. W. (2004). BASC-2: Behavior assessment system for children. Minneapolis: Pearson.

Rothbart, M. K. (2004). Commentary: Differentiated measures of temperament and multiple pathways to childhood disorders. Journal of Clinical Child and Adolescence Psychology, 33(1), 82-87. http://dx.doi. org/10.1207/S15374424JCCP3301_8

Rothbart, M. K. (2011). Becoming who we are: Temperament, personality and development. New York: Guilford Press.

Rothbart, M. K., Ahadi, S. A., Hershey, K. L., \& Fisher, P. (2001). Investigations of temperament at three to seven years: The children's behavior questionnaire. Child Development, 72(5), 1394-1408. http://dx.doi. org/10.1111/1467-8624.00355

Rothbart, M. K., \& Bates, J. E. (2006). Temperament. In W. Damon, R. M. Lerner, \& N. Eisenberg. Handbook of Child Psychology: Social, emotional and personality development (pp.99-165). New York: John Wiley \& Sons.

Rueda, M. R., Posner, M. I., \& Rothbart, M. K. (2011). Attentional control and self-regulation. In K. D. Vohs \& R. F. Baumeister (Eds.), Handbook of self-regulation: Research, theory, and applications (pp.284-299). New York: The Guilford Press.

Roy, A. K., Klein, R. G., Angelosante, A., Bar-Haim, Y., Leibenluft, E., Hulvershorn, L., ... Spindel, C. (2013). Clinical features of young children referred for impairing temper outbursts. Journal of Child and Adolescent Psychopharmacology, 23(9), 588-596. http://dx.doi.org/10.1089/cap.2013.0005 
Sameroff, A. (2009). The transactional model of development: How children and contexts shape each other. Washington, DC: American Psychological Association.

Sameroff, A. (2010). Dynamic developmental systems: Chaos and order. In G. W. Evans \& T. D. Wachs (Eds.), Chaos and its influence on children's development: An ecological perspective (pp.255-264). Washington, DC: American Psychological Association.

Sameroff, A. J., \& Chandler M. J. (1975). Reproductive risk and the continuum of caretaking casuality. In F. D. Horowitz, E. M. Hetherington, S. Scarr-Salapatek, \& G. M. Siegel (Eds.), Review of child development research (pp.187-244). Chicago, IL: University of Chicago Press.

Sameroff, A. J., \& Fiese B. H. (2000). Transactional regulation: The developmental ecology of early intervention. In J. P. Shonkoff \& S. J. Meisels (Eds.), Handbook of early childhood intervention (pp.135-59). New York: Cambridge University Press.

Sanders, M. R. (2008). Triple P: Positive Parenting Program as a public health approach to strengthening parenting. Journal of Family Psychology, 22(3), 506-517. http://dx.doi.org/10.1037/0893-3200.22.3.506

Schlotz, W., Jones, A., Godfrey, K., \& Phillips, D. (2008). Effortful control mediates associations of fetal growth with hyperactivity and behavioral problems in 7-to-9years-old children. Journal of Child Psychopathology and Psychiatry, 49(11), 1228-1236. http://dx.doi.org/ 10.1111/j.1469-7610.2008.01946.x

Silva, J. (2009). ACT: Raising Safe Kids Program. Washington, DC: American Psychological Association.

Sroufe, L. A. (1995). Emotional development: The organization of emotional life in the early years. New York: Cambridge University Press.
Stevenson, M., \& Crnic, K. (2013). Intrusive fathering children's self-regulation and social skills: A mediation analysis. Journal of Intellectual Disability Research, 57(6), 500-512. http://dx.doi.org/10.1111/j.1365-278 8.2012.01549.x

Vohs, K. D., \& Baumeister, R. F. (2011). Handbook of self-regulation: Research, theory, and applications. New York: The Guilford Press.

Vygotsky, L. S. (1996). A formação social da mente: o desenvolvimento dos processos psicológicos superiores. São Paulo: Martins Fontes.

Wachs, T. D., \& Evans, G. W. (2010). Chaos in context. In G. W. Evans \& T. D. Wachs (Eds.), Chaos and its influence on children's development: An ecological perspective (pp.3-13). Washington, DC: American Psychological Association.

Wang, Z., Deater-Deckard, K., Petrill, S. A., \& Thompson, L. A. (2012). Externalizing problems, attention regulation, and household chaos: A longitudinal behavioral genetic study. Development and Psychopathology, 24(3), 755-769. http://dx.doi.org/ 10.1017/S0954579412000351

Xu, Y., Farver, J. A. M., \& Zhang, Z. (2009). Temperament, harsh and indulgent parenting, and Chinese children's proactive and reactive aggression. Child Development, 80(1), 244-258. http://dx.doi.org/10.1111/j.14678624.2008.01257.x

Zhou, Q., Wang, Y., Deng, X., Eisenberg, N., Wolchik, S. A., \& Tein, J. (2008). Relations of parenting and temperament to Chinese children's experience of negative life events, coping efficacy, and externalizing problems. Child Development, 79(3), 493-513. http:// dx.doi.org/10.1111/j.1467-8624.2008.01139.x

Recebido: dezembro 8, 2014

Aprovado: janeiro 12, 2015 
